\title{
Refractory Malignant Male Reproductive System Neoplasm
}

National Cancer Institute

\section{Source}

National Cancer Institute. Refractory Malignant Male Reproductive System Neoplasm. NCI Thesaurus. Code C150534.

Malignant male reproductive system neoplasm that is resistant to treatment. 